\title{
Title: Determining the Mass of the Universe
}

Author(s):

Submitted to:

Authorss

Michael S. Warren (LANL, T-6)

Benjamin C. Bromley (LANL, T-6 and University of Utah)

Raymond Laflamme (LANL, T-6)

Wojciech H. Zurek (LANL, T-6)

John Salmon (California Institute of Technology)

Peter J. Quinn (European Southern Observatory)

DOE Office of Scientific and Technical Information (OSTI)

\section{Los Alamos

Los Alamos National Laboratory, an affirmative action/equal opportunity employer, is operated by the University of California for the U.S. Department of Energy under contract W-7405-ENG-36. By acceptance of this article, the publisher recognizes that the U.S. Government retains a nonexclusive, royaltyfree license to publish or reproduce the published form of this contribution, or to allow others to do so, for U.S. Government purposes. Los Alamos National Laboratory requests that the publisher identify this article as work performed under the auspices of the U.S. Department of Energy. Los Alamos National Laboratory strongly supports academic freedom and a researcher's right to publish; as an institution, however, the Laboratory does not endorse the viewpoint of a publication or guarantee its technical correctness. 


\section{DISCLAIMER}

This report was prepared as an account of work sponsored by an agency of the United States Government. Neither the United States Government nor any agency thereof, nor any of their employees, make any warranty, express or implied, or assumes any legal liability or responsibility for the accuracy, completeness, or usefulness of any information, apparatus, product, or process disclosed, or represents that its use would not infringe privately owned rights. Reference herein to any specific commercial product, process, or service by trade name, trademark, manufacturer, or otherwise does not necessarily constitute or imply its endorsement, recommendation, or favoring by the United States Government or any agency thereof. The views and opinions of authors expressed herein do not necessarily state or reflect those of the United States Government or any agency thereof. 


\section{DISCLAIMER}

Portions of this document may be illegible in electronic image products. Images are produced from the best available original document. 


\title{
Determining the Mass of the Universe
}

\author{
Michael S. Warren*, Raymond Laflamme, Wojciech H. Zurek, PECIV \\ Benjamin C. Bromley (LANL T-6/University of Utah), \\ John K. Salmon (Caltech), \\ Peter J. Quinn (European Southern Observatory). \\ DEC 132000 \\ OSTI
}

\begin{abstract}
The average mass density of the Universe, parameterized by $\Omega$ (Omega), is the most sought after single number in cosmology. It determines whether the Universe is open, expanding forever $(\Omega<1)$ or closed, eventually recollapsing $(\Omega>1)$. Unfortunately, after a half century of research $\Omega$ is still uncertain by at least a factor of five. Most of the mass is in the form of dark matter, and the precise relationship between its distribution and the distribution of the observable galaxies is still not known.

We have the developed tools that may significantly improve the measurements of the mass of the Universe. We have performed state-ofthe-art numerical simulations that provide complete dynamical information about both galaxies and dark matter, and our work has shown that the usual treatment of galaxies as point masses is unjustified. Additionally, we have proposed a method to determine the cosmic mass density from redshiftspace distortions induced by large-scale flows in the presence of nonlinear clustering.
\end{abstract}

\section{Background and Research Objectives}

In order to make progress in physical science where it is not possible to perform physical experiments, one usually attempts to isolate a small part of the hierarchy of structures and interactions, and to infer the fundamental physics from a specific model. The most important of the fundamental questions in cosmology is: How much mass is there in the Universe? We have made significant contributions to answering this question, by isolating and studying the behavior of clusters of galaxies, and developing advanced analyses of observational data.

Cosmological models of the expanding Universe are tightly constrained by the spectrum of density fluctuations discovered by the Cosmic Microwave Background Explorer satellite (COBE) and by the velocities of galaxies. However, a key parameter, $\Omega$, which specifies the average mass density in the Universe, remains to be measured accurately.

*Principal Investigator: msw@lanl.gov 
If $\Omega .>1$ then there is enough matter to "close" the observable Universe into a finite volume and to cause an ultimate collapse of space in a "Big Crunch."

For values of $\Omega<1$, the Universe is "open" and no collapse will occur. The delineating value, $\Omega=1$ (favored by inflationary models), corresponds to a flat (Euclidean) Universe whose expansion halts only at time $t=$ infinity.

New automated galaxy surveys, e.g., the Sloan Digital Sky Survey, are coming online to measure redshifts from millions of galaxies, probing the large-scale Universe at more recent epochs. Supercomputing advances are enabling simulations of the Universe to accurately track the physics of cosmological clustering over unprecedented range of scales, providing quantitative tests of cosmological models against the observed Universe.

Measurements of $\Omega$ come from observations of galaxies that move in an unknown sea of dark matter. The extraction of cosmological information from these observations has proved to be most difficult. However, with realistic simulations of the Universe, we can experiment on systems for which everything is known about the dynamics of dark matter and the galaxies. Our objective has been to give a proper theoretical treatment to the problem of galaxy motions in a field of dark matter and to optimize measures of $\Omega$ with the aid of high-quality numerical simulations and the ever-improving observations.

\section{Importance to LANL's Science and Technology Base and National R\&D Needs}

This project contributes directly to our institutional goals and objectives of fostering excellence in basic research, ensuring that our core science and technology capabilities are integrated and recognized by government, universities and industry as being among the nation's best, and fostering partnerships with universities and other government laboratories to enhance our research efforts and to extend our capabilities.

The software developed to perform the simulations required for this project have enhanced LANL's capability for modeling and high-performance computing, as well as served as a proving ground for high-performance computers. The main Laboratory benefit is the exploration at the forefront of basic science, which serves to enhance the Laboratory's visibility, credibility, and stature. 
The project has also helped our group to attract the best young computational physicists in the world to LANL, as well as demonstrating advanced techniques that are applicable to a variety of difficult fundamental problems in materials science, biology and chemistry.

\section{Scientific Approach and Accomplishments}

We modified our original approach toward measurements of $\Omega$ to include large-scale flows. With simulations we have optimized measurements of $\Omega$ from the statistical anisotropy in redshift surveys induced by large-scale flows (Bromley, Warren \& Zurek 1997). Fundamental to this effort was the development of more sophisticated algorithms for identifying galaxies in the simulations. The work of Pfitzner $(1997,2000)$ allowed us to accurately post-process the enormous multi-million particle datasets into mock galaxy catalogs suitable for further investigation.

We proposed a method to determine the cosmic mass density from redshift-space distortions induced by large-scale flows in the presence of nonlinear clustering. Nonlinear structures in redshift space, can contaminate distortions from linear flows on scales as large as several times the small-scale pairwise velocity dispersion. We work in the Fourier domain and propose a model to describe the anisotropy in the redshift-space power spectrum; tests with high-resolution numerical data demonstrate that the model is robust for both mass and biased galaxy halos on translinear scales and above. On the basis of this model, we propose an estimator of the linear growth parameter $=0.6 / b$, where $b$ measures bias (a measure of how clustered the galaxies are with respect to the underlying mass distribution), derived from sampling functions that are tuned to eliminate distortions from nonlinear clustering. The measure is tested on the numerical data and found to recover the true value of $b$ to within $10 \%$.

One of the simulations performed for this project won the 1997 Gordon Bell Prize for superior effort in practical parallel processing research (Warren \& Salmon 1997). Our treecode sustained 170 Gigaops over a continuous 9.4 hour period on 2048 nodes of the ASCI Red Teraops system, integrating the motion of 322 million mutually interacting particles for 288 timesteps, while saving over 100 Gbytes of raw data. Additionally, we have developed the capability to perform large parallel $\mathrm{N}$-body simulations locally on the Avalon cluster. 
We have continued to refine our data analysis capabilities to enable data mining from the very large datasets we are currently able to generate. This enables comparisons with the largest observational datasets such as the Las Campanas Redshift survey and the forthcoming Sloan Digital Sky Survey. A paper comparing our N-body code with the dozen leading cosmological codes in the world was published by the Astrophysical Journal. This paper shows that our numerical methods provided the highest resolution simulation among all of the participants. Another of the simulations performed for this project won the 1998 Gordon Bell Prize for superior effort in practical parallel processing research. This large parallel $\mathrm{N}$-body simulation was performed locally on the Avalon cluster (Warren et al. 1998). 


\section{Publications}

[1] Bromley, B.C., Warren, M.S., Zurek, W.H., "Estimating Omega from galaxy redshifts: Linear flow distortions and nonlinear clustering", Astrophysical Journal, 475, 414-420 (1997)

[2] Pfitzner, D. W., Salmon, J. K., Sterling, T., "Halo world: Tools for parallel cluster finding in astrophysical N-body simulations," Data Mining and Knowledge Discovery, 1, 419-438 (1997)

[3] Warren, M.S., Becker, D.J., Goda, M.P., Salmon, J.K., \& Sterling, T., "Parallel Supercomputing with Commodity Components , Proceedings of the International Conference on Parallel and Distributed Processing Techniques and Applications (PDPTA'97)", (1997)

[4] Warren, M.S., Salmon, J.K., "Pentium Pro Inside: I. A Treecode at 430 Gflops on ASCI Red, II. Price/Performance of \$50/Mflop on Loki and Hyglac, Supercomputing '97, IEEE Comp. Soc., (1997)

[5] Salmon, J. K. and Warren, M. S., "Parallel out-of-core methods for N-body simulation" In 8th SIAM Conf. on Parallel Processing for Scientific Computing, Philadelphia, SIAM, (1997).

[6] Warren, M. S., Germann, T. C., Lomdahl, P. S., Beazley, D. M., Salmon, J. K. "Avalon: An Alpha/Linux Cluster Achieves 10 Gflops for \$150k," In Supercomputing '98, Los Alamitos, IEEE Comp. Soc., (1998)

[7] Frenk, C. S., White, S. D. M., Bode, P. Bond, J. R., Bryan, G. L., Cen, R., Couchman, H. M. P., Evrard, A. E., Gnedin, N., Jenkins, A., Khokhlov, A. M., Klypin, A., Navarro, J. F., Norman, M. L., Ostriker, J. P., Owen, J. M., Pearce, F. R., Pen, U.-L., Steinmetz, M., Thomas, P. A., Villumsen, J. V., Wadsley, J. W., Warren, M. S., Xu, G., Yepes, G., "The Santa Barbara cluster comparison project: a comparison of cosmological hydrodynamics solutions," Astrophysical Journal, 525, 554-582 (1999)

[8] Warren, M. S., Avalon: An Alpha/Linux Beowulf cluster achieves unprecedented price/performance for Astrophysical simulations and data analysis., B.A.A.S, 30(4), (1999)

[9] Pfitzner, D. W., "The Structure of Halos in CDM Universes," Ph. D. Thesis, Australian National University, (2000)

[10] Tegmark, M., Bromley, B.C., "Observational evidence for stochastic biasing" Astrophysical Journal, 518(\#2/pt.2) L69-L72, (1999)

Figure 1: A rich cluster we have simulated with 5 million particles. This computation ran for 80 hours and required nearly 2 times $10^{\wedge}\{15\}$ floating point operations. Within the central object we have identified over 400 galaxies which can be used to directly model observational derivations of $\Omega$. 


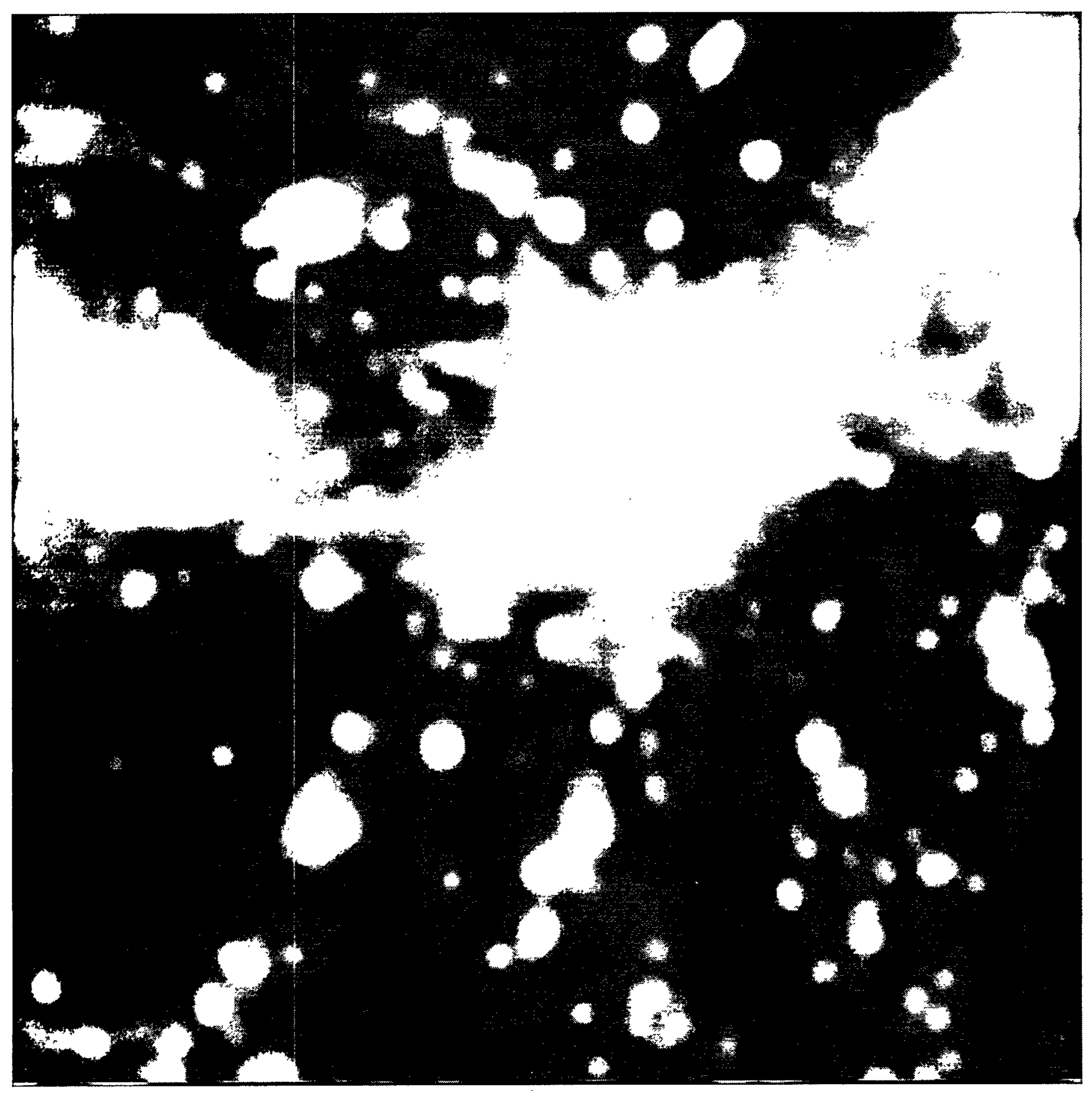

Figure 1 